\title{
Hybrid 2-port hand-assisted laparoscopic surgery for the 2-stage treatment of a complete bowel obstruction by distal sigmoid colon cancer: A case report
}

\author{
MASAYA MUKAI $^{1}$, KYOKO KISHIMA ${ }^{1}$, SHIN-ICHI IIZUKA ${ }^{1}$, HIROSHI FUKUMITSU ${ }^{1}$, \\ MAKI FUKASAWA ${ }^{1}$, NAOKI YAZAWA ${ }^{1}$, TAKAYUKI TAJIMA ${ }^{1}$, \\ MASATO NAKAMURA $^{2}$ and HIROYASU MAKUUCHI ${ }^{3}$ \\ ${ }^{1}$ Department of Surgery, Tokai University Hachioji Hospital, Ishikawa-cho 1838, Hachioji, \\ Tokyo 192-0032; Departments of ${ }^{2}$ Pathology, ${ }^{3}$ Surgery, Tokai University School of Medicine, \\ Bohseidai, Isehara, Kanagawa 259-1193, Japan
}

Received December 30, 2008; Accepted February 6, 2009

\section{DOI: $10.3892 /$ or_00000342}

\begin{abstract}
In July 2008, a 40-year-old man presented to his local physician with diffuse abdominal pain and severe abdominal distension. Impending bowel rupture due to colonic obstruction was strongly suspected. Complete obstruction of the distal sigmoid colon by a tumor was diagnosed, and emergency surgery was performed. A sigmoid colon loop colostomy was created within the range of subsequent resection to relieve the obstruction. After his general condition had improved and the risks were assessed, curative resection including removal of the stoma was performed by hybrid 2-port hand-assisted laparoscopic surgery. The tumor showed invasion of the serosa without lymph node metastasis, and its pathological diagnosis was stage II. Postoperatively, mild wound infection occurred at the hand access site (stoma), but it resolved with conservative treatment, and the patient was discharged on postoperative day 13. This case is reported here because of the good results.
\end{abstract}

\section{Introduction}

In recent years, less invasive laparoscopy-assisted colorectal surgery (LACS) has become very common. The indications for LACS cover a very wide range, from additional procedures

Correspondence to: Dr Masaya Mukai, Department of Surgery, Tokai University Hachioji Hospital, Ishikawa-cho 1838, Hachioji, Tokyo 192-0032, Japan

E-mail: mukai.masaya@hachioji-hosp.tokai.ac.jp

Abbreviations: LACS, laparoscopy-assisted colorectal surgery; HALS, hand-assisted laparoscopic surgery

Key words: sigmoid colon cancer, bowel obstruction, laparoscopyassisted colorectal surgery, hybrid hand-assisted laparoscopic surgery, 2-stage operation after endoscopic mucosal resection (EMR) of stage I early colorectal cancer to curative resection of stage II/III cancer and palliative surgery for advanced stage IV cancer (1-5). Unlike in the West, LACS in Japan does not usually involve hand-assisted laparoscopic surgery (HALS) or hybrid HALS combined with a standard procedure under direct vision (6-9). Instead, LACS is mainly performed with 5-6 ports including the camera port and a small incision of $35-45 \mathrm{~mm}(10,11)$. However, such pure LACS is difficult to perform in patients with complete bowel obstruction by left colon cancer or those who require combined resection due to invasion of other organs such as the urinary bladder or other parts of the intestine. Problems with the comparatively long operating time have also been pointed out (5). To solve these problems, we considered the use of hybrid 2-port HALS, in which HALS is combined with open surgery via a small incision $(45-55 \mathrm{~mm})$ as the hand access site $(5,12,13)$. Since hybrid 2port HALS is conducted with a small incision and only two ports, this represents a major difference from pure LACS using 5 to 6 ports. The features of hybrid 2-port HALS are as follows: i) palpation and tactile sensation ensure safe and reliable surgery; ii) protective and smooth procedures can be carried out by manual manipulation, particularly in patients with large and heavy tumors; iii) the procedure can be performed by only two surgeons; iv) it is an extension of standard operation, so the operating time is shorter; and v) there is only a shallow learning curve for the procedure $(5,13)$.

It has been reported that initial emergency surgery for complete obstruction of the left colon by cancer should be performed rapidly at any time with minimum invasion in order to relieve the high intraluminal pressure by creation of a safe and reliable temporary loop colostomy or ileostomy (12). We do not employ either of the conventional sites in the right transverse colon or ileum, but instead place a colostomy proximal to the obstruction within the range of resection at subsequent curative resection (12). After relief of the bowel obstruction, the dilation and edema resolve within $\sim 10$ days. When the general condition of the patient has improved sufficiently and work-up has been completed, elective surgery 


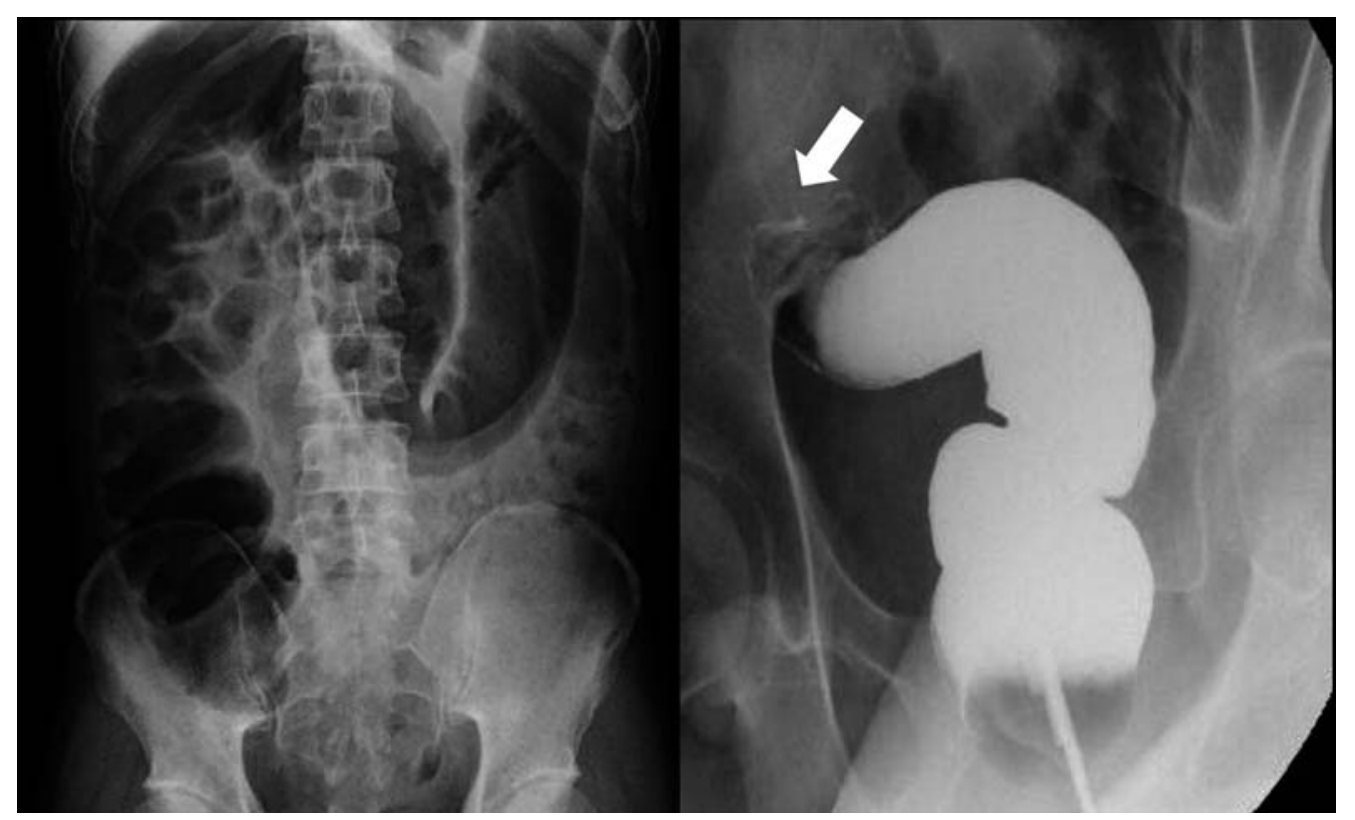

Figure 1. Plain abdominal X-ray film obtained on admission. Both the ascending colon and transverse colon showed marked dilatation to a diameter of $\sim 10 \mathrm{~cm}$ (left). Emergency gastrograffin enema shows marked stenosis of the distal sigmoid colon $\sim 25 \mathrm{~cm}$ from the anal verge, and complete obstruction was diagnosed (right, arrow).

can be performed safely. We created an emergency loop colostomy in the sigmoid colon proximal to the obstruction and within the range of subsequent resection in a patient with complete obstruction of the distal sigmoid colon by advanced colon cancer. After the general condition of the patient had improved, we performed curative resection including removal of the stoma by hybrid 2-port HALS, and good results were obtained as reported here.

\section{Case report}

The patient was a 40-year-old man with a history of good health. There was nothing relevant in his medical history, and he had no chronic diseases. From around April 2008, he occasionally felt a sharp pain in his lower abdomen, but he did not seek medical attention. In August 2008, diffuse abdominal pain and severe abdominal distention caused him to visit a local physician. A plain abdominal X-ray film suggested impending rupture of the bowel due to complete left colonic obstruction. He was referred to our hospital and was admitted as an emergency patient. The plain abdominal X-ray film obtained at admission showed marked dilatation of the ascending colon and transverse colon to a diameter of $\sim 10 \mathrm{~cm}$ (Fig. 1, left). Gastrograffin enema revealed marked stenosis of the distal sigmoid colon at $\sim 25 \mathrm{~cm}$ from the anal verge with complete obstruction (Fig. 1, right). The patient and his family were told that it was first necessary to immediately relieve the bowel obstruction by creation of a loop colostomy, and that after his general condition had improved and the risks and other factors had been adequately examined, curative resection would be performed as a second operation by LACS; hybrid 2-port HALS or standard laparotomy. After consent was obtained, emergency surgery was performed via a small midline subumbilical vertical incision $\sim 6 \mathrm{~cm}$ long taking into consideration the hand access site for subsequent surgery, and the stoma was formed near the top of the proximal sigmoid colon loop on the oral side of the obstruction within the range of subsequent resection (Fig. 2, left). When colonoscopy was performed later, the endoscope could not be passed through the stenosis, and biopsy of the lesion revealed moderately differentiated adenocarcinoma. Following adequate investigation and assessment of the risks after improvement of the ileus, hybrid 2-port HALS was performed for curative resection including the stoma at 11 days after the initial operation.

Findings at hybrid 2-port HALS. First, the loop colostomy near the top of the sigmoid colon on the oral side of the tumor was taken down, and a small midline laparotomy incision $\sim 6 \mathrm{~cm}$ long was made in the lower abdomen at the stoma site. Then, a hand access device (Lapdisc ${ }^{\circledR}, 120$ x 120 mm, Hakkou Co., Ltd., Nagano, Japan) was attached, and a 12-mm camera port and 5-mm surgical port were also inserted. The tumor was examined under pneumoperitoneum, and a careful intraperitoneal survey was performed (Fig. 3A). Mobilization and dissection of the sigmoid colon from the left paracolic gutter was performed by a lateral approach, and the left ureter was identified (Fig. 3B). The hypogastric plexus was identified on the mainly located anterior surface of the descending aorta between the aorta and inferior mesenteric artery/vein (IMA/V), as dissection was continued from the IMA/V through the pelvic floor. After careful manipulation, complete preservation of the plexus was carried out for maintenance of sexual function, especially ejaculation. A large fenestration was created in the sigmoid mesocolon as far as the anterior surface of the aorta/sacrum, so that the sigmoid mesocolon/ sigmoid colon could be easily pulled out of the peritoneal cavity. Then the left colic artery/vein was identified at their origins from the IMA/V under direct vision, and D2 dissection was performed distal to the left colic vessels with a safety 


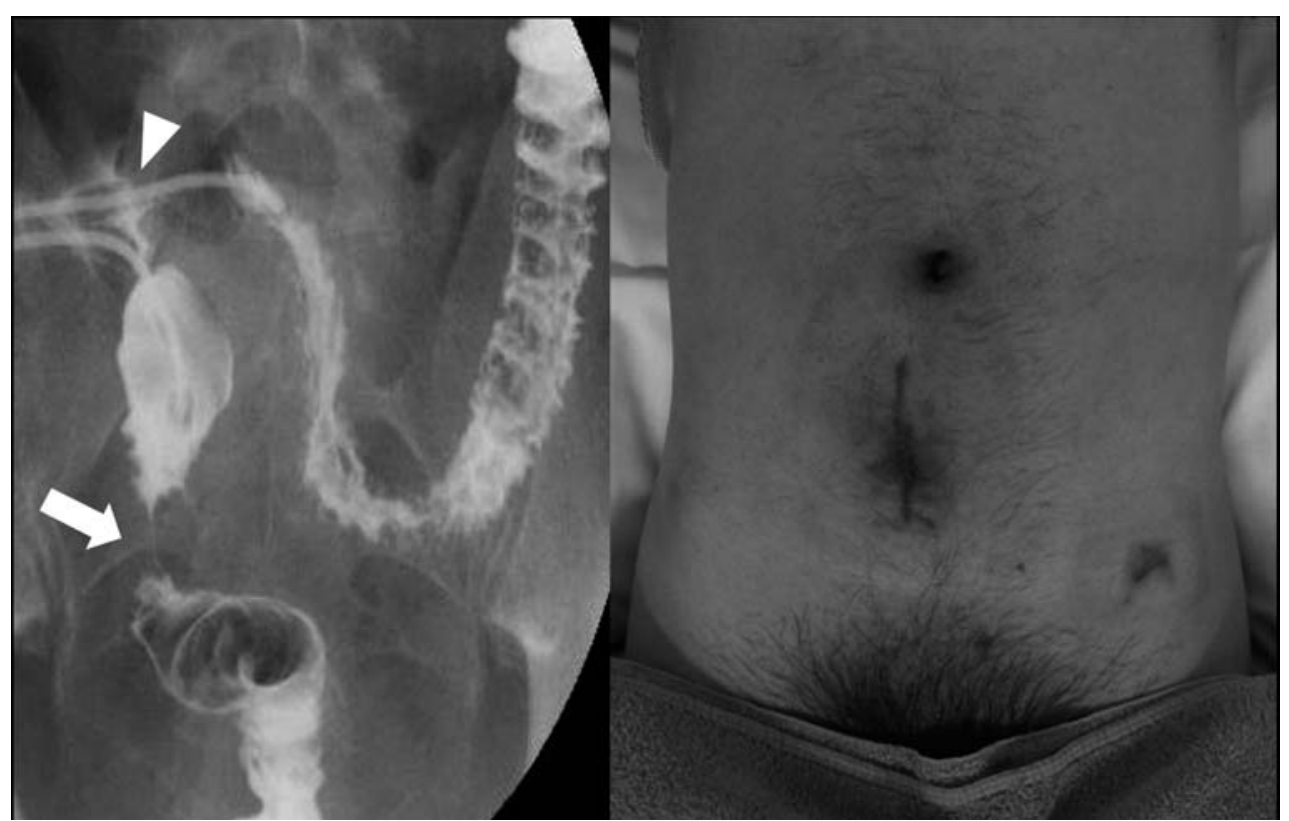

Figure 2. The tumor was located in the distal sigmoid colon (left, arrow). To relieve the obstruction and improve the general condition, a double-barreled loop sigmoid colostomy was formed near the middle of the sigmoid colon (left, arrowhead). An overall view of the abdominal wounds is shown at 5 months after hybrid 2-port HALS (right).

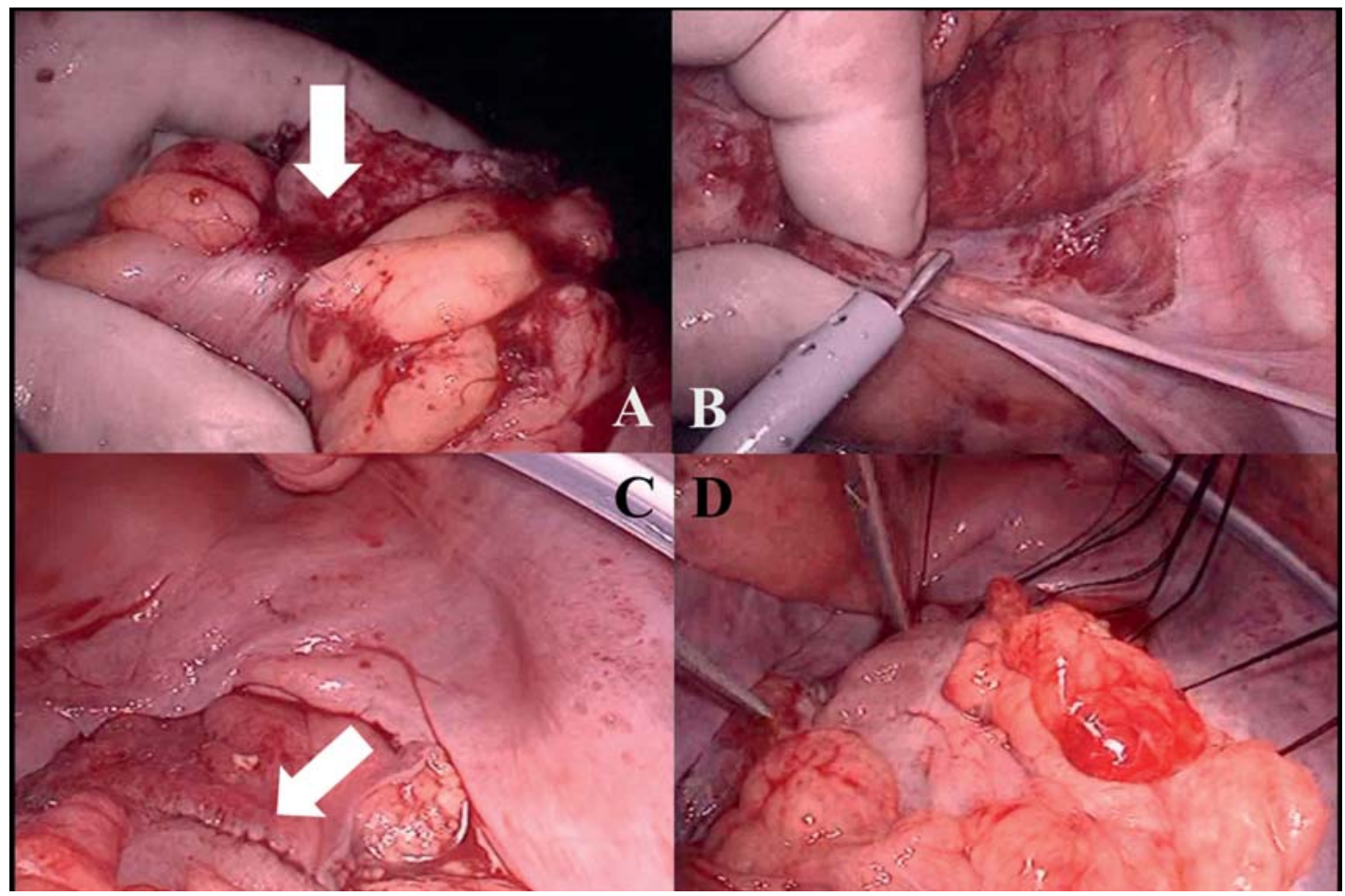

Figure 3. The tumor was a mass the size of a hen's egg in the distal sigmoid colon associated with marked serosal dimpling (A, arrow). Mobilization and dissection of the sigmoid colon from the left paracolic gutter was performed by a lateral approach (B). Then D2 resection was performed, and intraperitoneal anastomosis was carried out by the standard double stapling technique $(\mathrm{C}$, arrow). The area up to the junction between the pelvic peritoneum and bladder was closed with manual sutures (D).

margin of at least $5-10 \mathrm{~cm}$ on the oral and anal sides of the tumor. The rectum was completely transected in one step using a 51-mm curved cutter (green cartridge, Ethicon EndoSurgery, Puerto Rico, USA). Before anastomosis, closure of the sigmoid mesocolon and mesorectum was completed at the resection site. Then standard double stapling of the colon and rectum was performed intraperitoneally with a Proximate ILS Endopath Endo-circular stapler (ECS33; Ethicon EndoSurgery) (Fig. 3C). Complete closure as far as the junction of the pelvic peritoneum and bladder was also performed by 


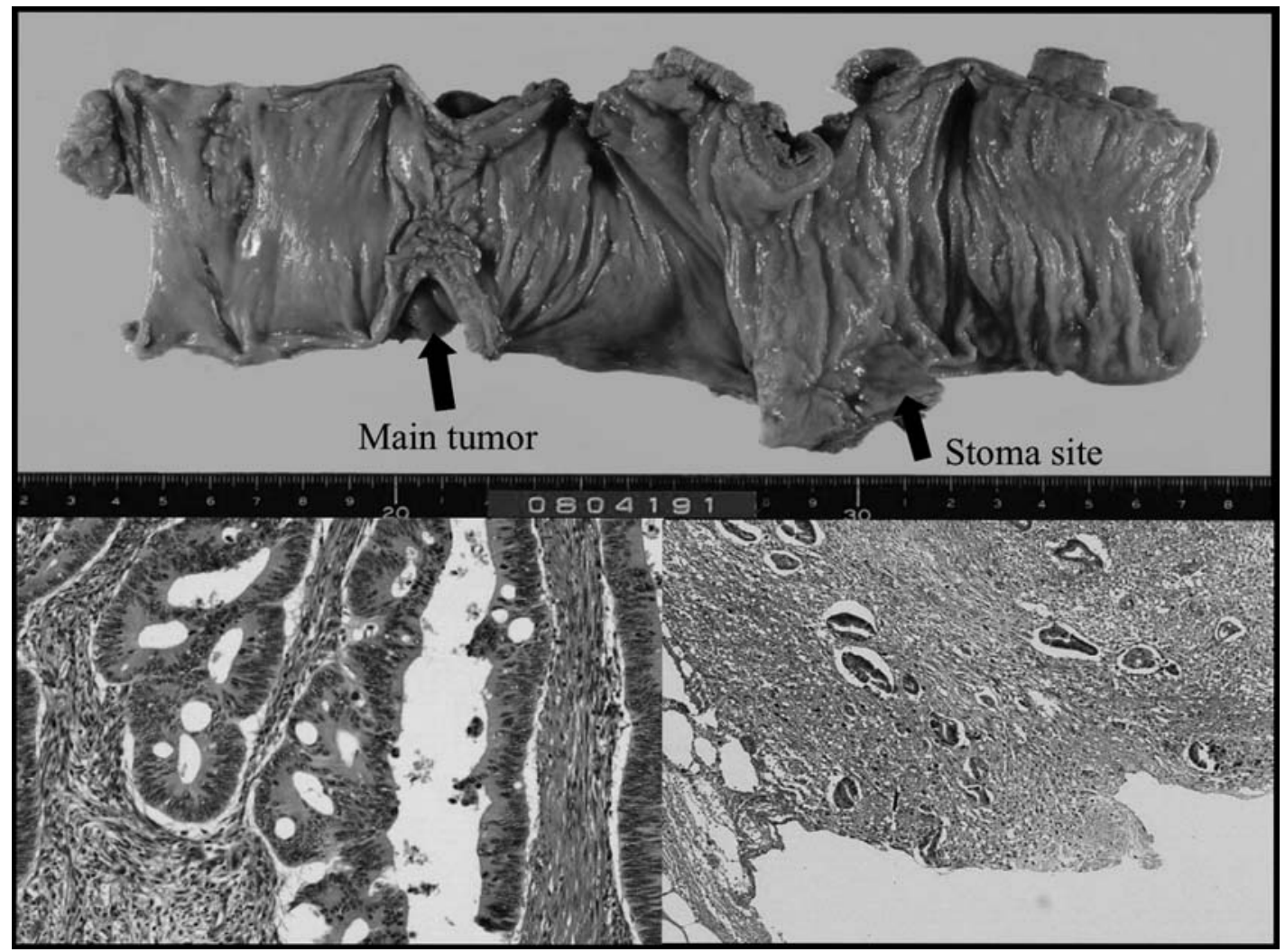

Figure 4. The sigmoid colon cancer was found to be POHONO (0/24) with serosal exposure (SE) and was a moderately differentiated adenocarcinoma in stage II upon histopathological diagnosis.

manual suturing (Fig. 3D). After hemostasis was confirmed and intraperitoneal lavage was performed, a drain tube was passed through the $12-\mathrm{mm}$ port, the hand access site was closed, and the operation was completed.

The tumor was a moderately differentiated adenocarcinoma surgically classified as $\mathrm{POHONO}(0 / 24)$ with serosal exposure (SE) and was in stage II histopathologically (Fig. 4). Postoperatively, a mild wound infection occurred, but it resolved with conservative treatment. The patient had no sexual dysfunction, especially ejaculatory disorders or retrograde ejaculation. The postoperative course was good, and he was discharged on day 13 after the second procedure (day 24 after admission) (Fig. 2, right).

\section{Discussion}

In patients with complete bowel obstruction caused by cancer of the left colon, particularly tumors of the descending colon or sigmoid colon, transanal drainage is difficult, and emergency surgical decompression is often required (14-16). When curative resection of the tumor, including lymph node dissection, is performed at the initial operation, it is almost always necessary to fashion a temporary loop ileostomy or right transverse colostomy in addition to performing resection of the primary tumor and anastomosis (17-19). Primary direct anastomosis with massive intraoperative irrigation from the oral side of the tumor does not require a temporary colostomy, but such methods have the major disadvantages of a relatively long operating time and excessive invasiveness for emergency surgery in elderly patients (20-22). There have also been reports of complications such as intraoperative rupture of dilated bowel, wound infection, suture dehiscence, severe peritonitis and DIC $(23,24)$. We always reduce the intraluminal pressure first by creating a loop colostomy within the range of subsequent curative resection in patients with complete bowel obstruction by left colon cancer. After the general condition of the patient has improved and adequate work-up has been completed, curative resection of the tumor with removal of the stoma is performed as an elective surgery $(5,12)$. This method involves two-stage surgery, but it has the advantage of only one anastomosis, as well as no effect on curability, and no complications such as anastomotic leakage or stenosis associated with closure of the stoma.

In Japan, pure LACS is widely performed as minimally invasive surgery, but there are problems such as the small number of surgeons familiar with LACS and a shortage of suitable anesthesiologists. Even in relatively large hospitals, LACS has not become the gold standard, and surgeons at some institutions are no longer performing routine LACS. Since pure LACS requires the surgeon and first assistant to use four sets of forceps, at least three experienced surgeons including one for the camera are needed. The main problems with LACS are as follows: i) tactile sensation is poor; ii) it is difficult to remove large and heavy tumors; iii) it is difficult to assess the overall operating field; iv) procedures are mainly performed with the laparoscope so the operating time increases; and v) a technical certification system for laparoscopic surgery exists in Japan and it takes a long time to gain enough experience. Hybrid 2-port HALS differs from pure LACS in various respects. Procedures such as ligation 
and ablation of vessels like the main colic artery/vein with lymph node dissection, anastomosis, and closure of the mesocolon/mesorectum after colorectal resection can be performed relatively easily under direct vision like standard laparotomy, and it can be performed safely in patients with ileus such as the present case or patients with tumors invading other organs $(5,12,13)$.

In recent years, the indications for LACS have been based on different standards at each institution, covering a very wide range from stage I to stage IIIa (N1 lymph node metastasis involving $\leq 3$ nodes) or stage IIIb tumors, or even palliative operations for stage IV cancer (1-5). In Western countries, radiation therapy is often administered concomitantly to patients with advanced lower rectal cancer (25-27). In Japan, there has been a long-standing debate about the value of N3 lymph node dissection around the internal and external iliac vessels and bilateral lateral lymph node dissection rather than radiation therapy (28-30). There have been many reports that the prognosis is improved when patients with lymph node metastasis undergo lateral lymph node dissection, but prophylactic bilateral lateral lymph node dissection remains controversial, and no definite conclusions have been established (28-30). Therefore, LACS is also performed in a wide range of rectal cancer patients without obvious lateral lymph node metastasis preoperatively $(5,13)$. When hybrid 2port HALS is performed as elective surgery, if the posterior surface/anal side of the tumor is held in the left hand, there are no major differences with regard to tumor dissection from the procedures employed for standard laparotomy. It is also possible to perform procedures at the pelvic floor and the lower part of the bladder or posterior surface of the prostate, sites that cannot be observed at conventional laparotomy, using a monitor together with magnification from the laparoscope. In almost all of these patients, LACS; hybrid 2-port HALS is indicated. The usual indications for hybrid 2-port HALS include i) patients who can tolerate general anesthesia for 2 hours, ii) patients with no major concomitant diseases such as cardiac or pulmonary conditions, and iii) if possible, a P.S. of $0-1$, an age $\leq 75$ years, and no evidence of lateral lymph node metastasis in patients with advanced rectal cancer $(5,13)$. In patients with advanced lower rectal cancer, a transverse incision of $45-55 \mathrm{~mm}$ is usually made at the upper margin of the pubic bone, but open lateral lymph node dissection in patients with nodal metastases can be achieved by making a small incision for hand access such as a lower abdominal incision or lower abdominal pararectal incision in the vicinity of the metastatic lymph nodes. In the future, it will be necessary to perform a detailed comparison with standard laparotomy in terms of recurrence, including intrapelvic local recurrence and distant metastasis, by investigating more patients including those with advanced lower rectal cancer.

\section{Acknowledgements}

This study was supported by grants from the Occult Neoplastic Cell Research and Study Group (\#2008-5007; Tokai University Hachioji Hospital, Tokyo, Japan) and the Research and Study Program of Tokai University Educational System General Research Organization (\#2007-04; Tokai University School of Medicine, Kanagawa, Japan).

\section{References}

1. Mukai M, Tokunaga N, Ishida H, Makuuchi H, Tajima $\mathrm{T}$ and Mitomi T: Clinical experiences with laparoscopic colectomy. Dig Endosc 9: 11-15, 1997.

2. Mukai M, Okamoto Y, Oida Y, Mukoyama S, Ito I, Nakasaki H, Kawai K, Sato S and Makuuchi H: Endoscopic mucosal resection for superficially spreading colonic neoplasms larger than $5 \mathrm{~cm}$ in the right colon after dilute sodium hyaluronate injection: Report of two cases. Endoscopy 35: 973-975, 2003.

3. Makuuchi H and Sugihara K: Knacks \& Pit Falls: Surgery of the Colon, Rectum and Anus. 2nd edition, Bunkoudou, Co., Ltd., Tokyo, 2004.

4. Guideline of Large Bowel Cancer in Japan. Japanese Society for Cancer of the Colon and Rectum, Tokyo, 2005.

5. Mukai M, Tanaka A, Tajima T, Yamagiwa T, Okada K, Fukasawa M, Sato K, Oida Y, Nakamura M and Makuuchi H: Two-port hand-assisted laparoscopic surgery for the 2-stage treatment of a complete obstruction by left colon cancer: A case report. Oncol Rep 19: 875-879, 2008.

6. Romanelli JR, Kelly JJ and Litwin DE: Hand-assisted laparoscopic surgery in United States: an overview. Semin Laparosc Surg 8: 96-103, 2001

7. Nakajima K, Lee SW, Cocilovo C, Foglia C, Kim K, Sonoda T and Milson JW. Hand-assisted laparoscopic colorectal surgery using GelPort. Surg Endosc 18: 102-105, 2004.

8. Nakajima K, Lee SW, Cocilovo C, Foglia C, Sonoda T and Milson JW: Laparoscopic total colectomy: hand-assisted vs. standard technique. Surg Endosc 18: 582-586, 2004.

9. Yamaguchi Y, Minami K, Kawabuchi Y, Emi M and Toge T: Anterior resection of rectal cancer through a one hand-size incision with or without laparoscopy: proposal of one handsize incision surgery (OHaSIS). J Surg Res 129: 136-141, 2005.

10. Jacobs M, Verdja JC and Goldstein HS: Minimally invasive colon resection (laparoscopic colectomy). Surg Laparosc Endosc 1: 144-150, 1991.

11. The Clinical Outcomes of Surgical Therapy Study Group. A comparison of laparoscopically assisted and open colectomy for colon cancer. N Engl J Med 350: 2050-2059, 2004.

12. Mukai M, Himeno S, Mukoyama S, Tajima T, Saito Y, Ito I, Nakasaki H, Sato S and Makuuchi H: Is the temporally loop colostomy in the right transverse colon appropriate for complete obstruction by colorectal cancer? Oncol Rep 10: 693698, 2003.

13. Mukai M, Fukasawa M, Kishima K, Iizuka S, Fukumitsu H, Yazawa N, Tajima T, Nakamura M and Makuuchi H: Transanal reinforcing sutures after double stapling for low rectal cancer: Report of two cases. Oncol Rep 21: 335-339, 2009.

14. Hoffmann $J$ and Jensen HE: Tube cecostomy and staged resection for obstructing carcinoma of the left colon. Dis Colon Rectum 27: 24-32, 1984.

15. Mackenzie S, Thompson SR and Baker LW. Management options in malignant obstruction of the left colon. Surg Gynecol Obstet 74: 337-344, 1992.

16. Chen HS and Sheen-Chen SM: Obstruction and perforation in colorectal adenocarcinoma: An analysis of prognosis and current trends. Surgery 127: 370-376, 2000.

17. Williams NS, Nasmyth DG, Jones D and Smith AH: Defunctioning stomas: a prospective controlled trial comparing loop ileostomy with loop transverse colostomy. Br J Surg 73: 566-570, 1986

18. Sjödahl R, Franzen T and Nyström PO: Primary versus staged resection for acute obstructing colorectal carcinoma. Br J Surg 79: 685-688, 1992.

19. Rullier E, Le Toux N, Laurent C, Garrelon J-L, Parneix M and Saric J: Loop ileostomy versus loop colostomy for defunctioning low anastomoses during rectal cancer surgery. World J Surg 25: 274-278, 2001.

20. Stothert JC Jr, Brubacher L and Simonowitz DA: Complications of emergency stoma formation. Arch Surg 120: 1145-1147, 1985.

21. Thomson WH and Carter SS: On-table lavage to achieve safe restorative rectal and emergency left colonic resection without covering colostomy. Br J Surg 73: 61-63, 1986.

22. Pollock AV, Playforth MJ and Evans M: Peroperative lavage of the obstructed left colon to allow safe primary anastomosis. Dis Colon Rectum 30: 171-173, 1987.

23. Porter JA, Salvati EP, Rubin RJ and Eisentat TE: Complications of colostomies. Dis Colon Rectum 32: 299-303, 1989. 
24. Karanjia ND, Corder AP, Holdsworth PJ and Heald RJ: Risk of peritonitis and fatal septicaemia and the need to defunction the low anastomosis. Br J Sur 78: 196-198, 1991.

25. NIH Consensus Conference: Adjuvant therapy for patients with colon and rectal cancer. JAMA 264: 1444-1450, 1990.

26. Krook JE, Moertel CG, Gunderson LL, Wieand HS, Collins RT, Beart RW, Kubista TP, Poon MA, Meyers WC, Mailliard JA, Twito DI, Morton RF, Veeder MH, Witzig TE, Cha S and Vidyarthi SC: Effective surgical adjuvant therapy for high-risk rectal carcinoma. N Engl J Med 324: 709-715, 1991.

27. Wolmark N, Wieand HS, Hyams DM, Colangelo L, Dimitrov NV, Romond EH, Wexler M, Prager D, Cruz AB Jr, Gordon PH, Petrelli NJ, Deutsch M, Mamounas E, Wickerham DL, Fisher ER, Rockette H and Fisher B: Randomized trial of post operative adjuvant chemotherapy with or without radiotherapy for carcinoma of the rectum: National Surgical Adjuvant Breast and Bowel Project Protocol R-02. J Natl Cancer Inst 92: 388396, 2000.
28. Moreira LF, Hizuta A, Iwagaki H, Tanaka N and Orita K: Lateral lymph node dissection for rectal carcinoma below the peritoneal reflection. Br J Surg 81: 293-296, 1994.

29. Hida J, Yasutomi M, Fujimoto K, Maruyama T, Okuno K and Shindo K: Does lateral lymph node dissection improve survival in rectal carcinoma? Examination of node metastases by the clearing method. J Am Coll Surg 184: 475-480, 1997.

30. Sato H, Maeda K, Maruta M, Matsumori K and Koide Y: Who can get the beneficial effect from lateral lymph node dissection for Dukes C rectal carcinoma below the peritoneal reflection? Dis Colon Rectum 49: 3-12, 2006. 